\title{
Tactile-based Manipulation of Deformable Objects with Dynamic Center of Mass
}

\author{
Mohsen Kaboli ${ }^{1}$, Kunpeng Yao $^{1}$, and Gordon Cheng ${ }^{1}$
}

\begin{abstract}
Tactile sensing feedback provides feasible solutions to robotic dexterous manipulation tasks. In this paper, we present a novel tactile-based framework for detecting/correcting slips and regulating grasping forces while manipulating deformable objects with the dynamic center of mass. This framework consists of a tangential force based slip detection method and a deformation prevention approach relying on weight estimation. Moreover, we propose a new strategy for manipulating deformable heavy objects. Objects with different stiffnesses, surface textures, and centers of mass are tested in experiments. Results show that proposed approaches are capable of handling objects with uncertainties in their characteristics, and also robust to external disturbances.
\end{abstract}

\section{INTRODUCTION AND RELATED WORK}

Recent advances in robotic hands have simplified the problem of grasping known rigid objects [1]. However, successfully grasping and manipulating of an unknown object with uncertainty in its characteristics such as surface texture [2][5] and center of mass is still difficult for robotic systems. Tactile sensing enables robotic systems to interact safely with humans and surrounded objects [6], [7]. Tactile feedback provides feasible solutions to slip detection problem, which is crucial to regulate grasping force in robotic dexterous manipulation. Slip signals can be detected in several ways. One common approach is to detect vibrations on the contact surface. Engeberg et al. [8] filtered force signals from strain gauges through several band-pass filters with different frequencies and then transformed using FFT. Teshigawara et al. [9] developed a novel sensor which can detect slip by sensing high-frequency signal components above $1 \mathrm{kHz}$. Schoepfer et al. [10] used Piezo-Resistive Tactile Sensor and a frequency-domain approach for incipient slip detection. However, our work is novel in employing the OptoForce sensors for these tasks, which provides the robotic system with a wide frequency range and increased sensitivity over traditional tactile sensors. Although vibration based methods are useful for detecting slip signals, their effectiveness could be gravely impaired due to the vibration generated by robotic systems, especially in the case of manipulating/rotating objects with dynamic centers of mass. Slip signals can also be detected by estimating friction cone, i.e. by following Coulomb's law of friction. This requires the calculation of friction coefficient $\mu$ on the contact surface. In order to maintain a stable grasp, the ratio of exerted tangential force

\footnotetext{
${ }^{1}$ Mohsen Kaboli, Kunpeng Yao, and Gordon Cheng are with the Institute for Cognitive Systems, Faculty of Electrical Engineering and Information Technology, Technical University of Munich (TUM), Germany. Email: mohsen.kaboli@tum.de. Video to this paper: http://web.ics.ei.tum.de/ mohsen/videos/Humanoids2016.mp4
}

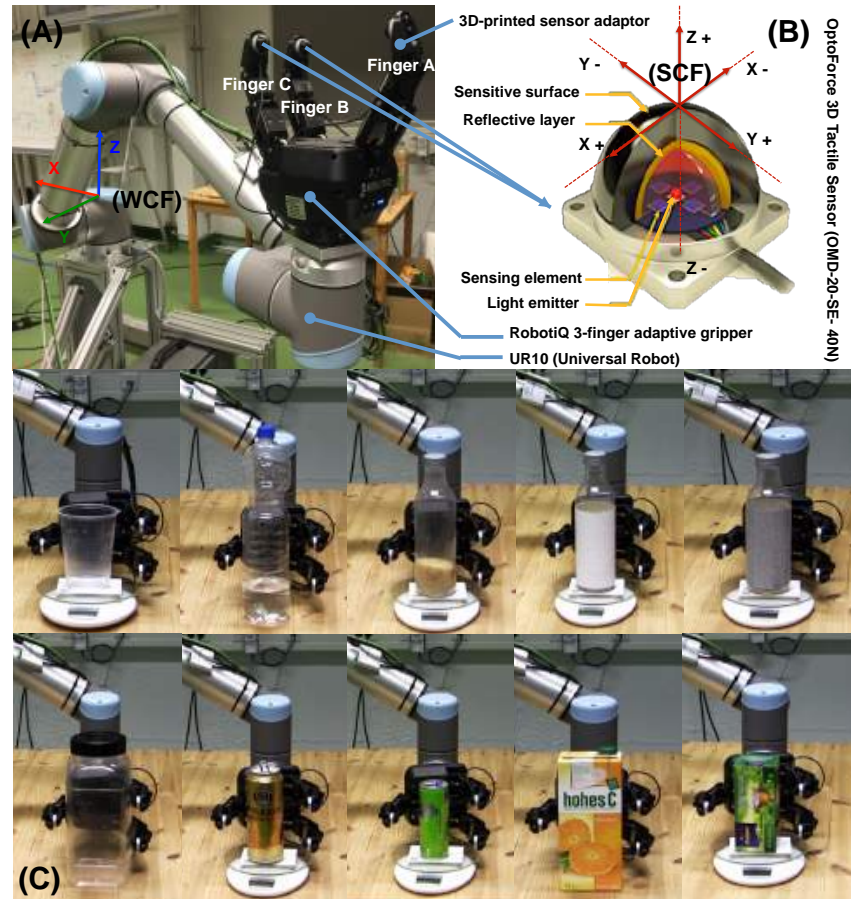

Fig. 1. Three-finger adaptive robot gripper from Robotiq is equipped with OptoForce sensors (B) and mounted on the UR10 robotic arm (A). Experimental objects are shown in $(\mathrm{C})$.

to normal force should be maintained within the friction cone [11]. Kobayashi et al. [12] used force/torque sensors to measure pressure distribution in order to detect slip. Instead of increasing grasping force, they propose to increase the number of applied fingers to stop slip. However, this method is not adaptable for dynamic manipulation of objects, and the relationship of multi-fingers was not considered, which may influence the grasping stability. In this study, we regulate grasping force and adjust relative positions of fingers, in order to realize dynamic manipulation tasks with a threefinger gripper. Some related studies also include approach to distinguish linear slip and rotational slip [13], and approach to discriminate between finger/object and object/world slip events, relying on multidimensional coherence measuring [14]. The performance of slip detection methods, including band pass filter, friction cone, and machine learning approaches are compared in [15]. Since slip signal reflects the stability of grasping, it plays the role of error feedback for the robotic system in manipulation tasks. The robotic system should react promptly to slip signals in order to maintain a stable manipulation. Su et al. [16] detected slip using two methods, including measuring the reduction of normal force as well as detecting micro-vibration on the sensor skin. A 
slip-detection based controller was proposed for simple pickand-place tasks. Here we use tangential force rather than normal force to detect slip.

Contribution: In this paper, we present a novel tactile based framework for correcting slips and regulating grasping forces in dynamic manipulation tasks, which is independent of the grasped object's characteristics, such as stiffness, surface texture, and center of mass. This framework consists of a tangential-force based slip detection method to correct slip and a deformation prevention approach to regulate grasping force, which adjusts the relative positions of fingers in real time, and is realized by estimating the weight of the grasped object. Furthermore, we propose a new strategy for manipulating deformable heavy objects by changing their poses.

\section{SYSTEM DESCRIPTION}

Robotic System: A Robotiq three-finger adaptive gripper was installed at the end of a 6-DoF UR10 (Universal Robots) robotic arm. The gripper is under-actuated (see Fig. 1-A), and its Finger A pushes against Finger B and Finger C. The position range of each finger is divided into 255 counts, with 0 indicating fully open, and 255 the fully closed. In this paper, we represent the position of each finger using counts.

Tactile Sensors: We used OptoForce OMD-20-SE-40N $3 \mathrm{D}$ force sensor set. The OptoForce sensor can measure forces exerted on the contact surface in three directions with a wide range of measurement. In this paper, we discuss forces in two coordinate frames: world coordinate frame (WCF) (see Fig. 1-A) and sensor coordinate frame (SCF) (see Fig. 1B). In SCF, we discuss the tangential force vector $\boldsymbol{f}_{T_{i}}$ and the normal force vector $\boldsymbol{f}_{N_{i}}$ exerted on the grasped object, with norms $\left|\boldsymbol{f}_{T_{i}}\right|$ and $\left|\boldsymbol{f}_{N_{i}}\right|$. The force vectors in WCF are represented as $\boldsymbol{f}_{x_{i}}, \boldsymbol{f}_{y_{i}}$, and $\boldsymbol{f}_{z_{i}}$, with corresponding norms $\left|\boldsymbol{f}_{x_{i}}\right|,\left|\boldsymbol{f}_{y_{i}}\right|$, and $\left|\boldsymbol{f}_{z_{i}}\right|$. The subscript $i$ denotes the number of finger $(i=1,2,3)$, and $P_{i}$ is the corresponding finger position. The weight of an object of mass $m$ is calculated as $\boldsymbol{W}=m \boldsymbol{g}$, with $\boldsymbol{g}$ being the gravitational acceleration.

Experimental Objects: We selected 10 everyday objects with deformable surfaces and various materials, including a disposable cup, a deformable bottle filled with $300 \mathrm{~mL}$ water, a plastic bottle with three different surface textures (texture 1: uniform meshy texture; texture 2: non-uniform rough texture; texture 3: smooth texture), each of which is filled with $200 \mathrm{~g}$ rice, a large flexible plastic jar, a golden aluminum can and a green aluminum can with different stiffnesses, a juice container and a tea box, both of which are made of cardboard with different stiffnesses (see Fig. 1-C).

\section{Methodology}

In this section, we first propose our approach to reduce the deformation of grasped objects due to the applied large grasping. Then, we summarized our proposed approaches into a framework to realize real-time control of the gripper. In addition, we suggest a novel strategy for preventing slip of deformable heavy objects.

\section{A. Deformation Prevention}

Grasped object deforms as grip force increases. Since the movement direction of the gripper's fingers is perpendicular to the local contact surface, normal force serves as an indicator of deformation extent. Therefore, to avoid any large local deformation of objects, an upper bound for normal force, $\bar{f}_{N}$, is required for all fingers according to the stiffness of target object. Moreover, as fingers of the gripper may share different portions of grasping force due to irregularity of the object's shape, it is necessary to adjust the relative positions of the fingers. In this regard, we suggest to estimate the weight of the grasped object. We decompose the force exerted by each finger in WCF and calculate the resultant force along $Z_{W C F}$ direction, $\left|\sum_{i} \boldsymbol{f}_{z_{i}}\right|$, as an estimation of the grasped object's weight as long as the grasp is stable, i.e. neither slip nor large deformation occurs. In an ideal critical stable state, resultant force in $Z_{W C F}$ direction should exactly balance the object's weight $|\boldsymbol{W}|$; however, considering the impulse generated by the varying center of mass, a margin should be taken into consideration. Hence, if the norm of current resultant force has exceeded a certain percentage over $|\boldsymbol{W}|$, the gripper is considered to have applied redundant forces, which should be reduced. Since the target object's weight is estimated only in stable state, this suggested approach can also be used for object whose weight varies during manipulation.

\section{B. Slip Correction and Force Regulation Framework}

Here we propose a position-level slip correction and grip force regulation framework. On the one hand, it regulates grasping force to prevent objects from sliding. We detect slip when it happens for the first time by measuring the increasing rate of tangential force, i.e. a slip signal is detected as soon as the increment of $\boldsymbol{f}_{T}$ has exceeded a certain percentage $\delta$ within a short time period $\Delta t$. As soon as the first slip is detected, the ratio of the tangential force's norm $\left|\boldsymbol{f}_{T}\right|$ to the normal force's norm $\left|\boldsymbol{f}_{N}\right|$ is calculated as an estimation of the friction coefficient $\mu_{i}$ on the corresponding contact area, which will be used to prevent slip during the dynamic manipulation process, i.e. a slip is detected as soon as $\left|\boldsymbol{f}_{T_{i}}\right| /\left|\boldsymbol{f}_{N_{i}}\right| \geq \mu_{i}$ and then grip force is supposed to be increased to stop slip. On the other hand, large deformation of grasped object is prevented not only by maintaining normal force exerted by each finger within its corresponding upper bound, but also by adjusting the resultant force in $Z_{W C F}$ direction, w.r.t. the estimated weight. This framework is summarized in Algorithm 1. Notice that $\left|\boldsymbol{f}_{T_{i}}(t)\right|$ denotes the value of $\boldsymbol{f}_{T_{i}}$ sensed at time $t$. After initialization, gripper closes to contact the target object. All fingers are controlled individually, so each finger stops closing as soon as its exerted normal force exceeds $f_{N_{\epsilon}}$. Hence, the grasping gesture can adapt the shape of the target object's surface. Once target object is grasped, the robotic arm starts lifting the grasped object up. In the meantime, if slip happens, $\mu_{i}$ is calculated for each contact area. Then, the gripper closes its fingers for one position count to stop slip and $|\boldsymbol{W}|$ is estimated. However, if the target object is lifted up without slip, the 


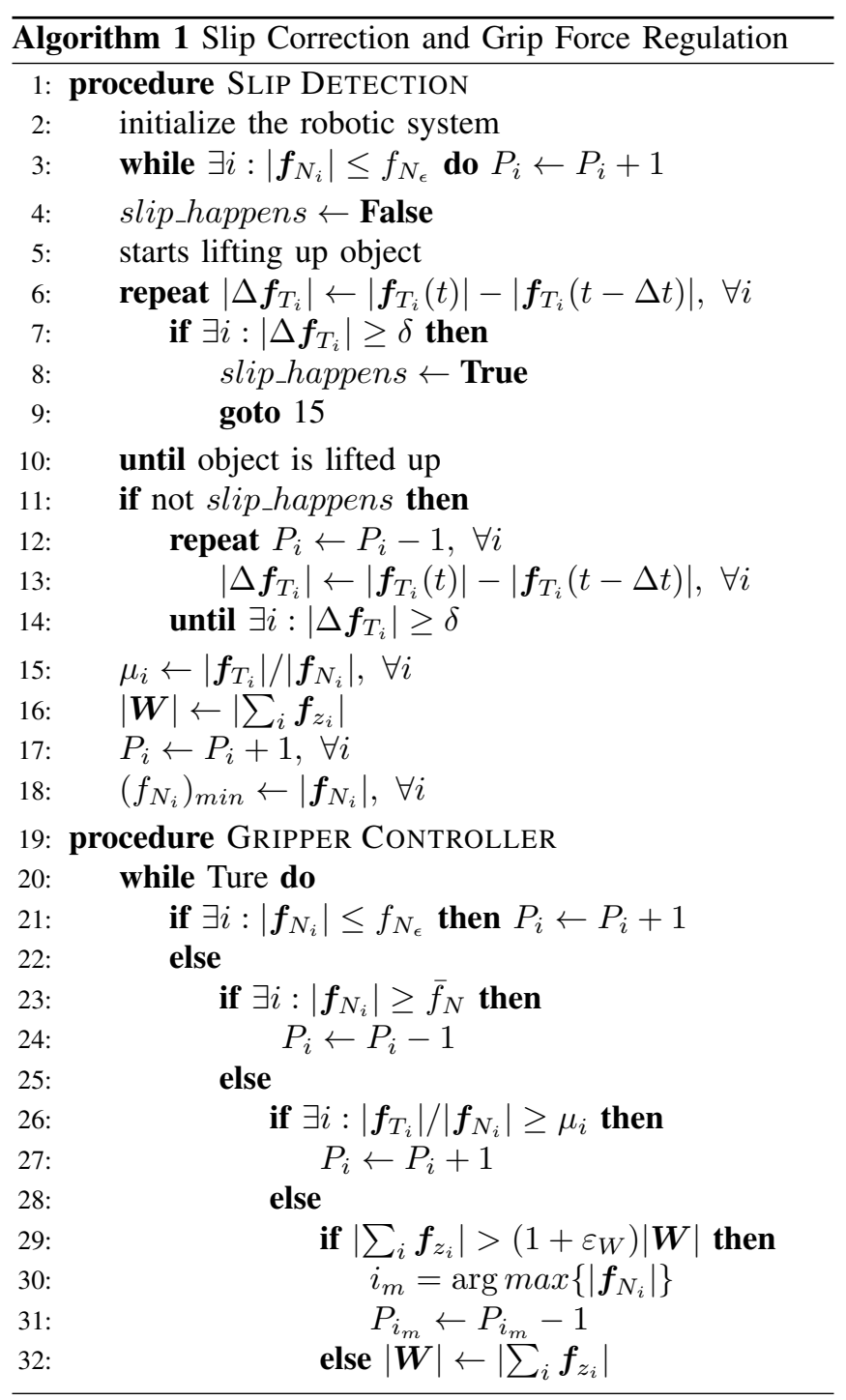

gripper will keep releasing all its fingers simultaneously for one position count at each time, until one slip happens and is detected. Afterwards, dynamic manipulation process will start. In each control loop, contact state (touch or not) of each finger will be checked firstly to ensure contact. Then the exerted normal force and force ratio $\left|\boldsymbol{f}_{T_{i}}\right| /\left|\boldsymbol{f}_{N_{i}}\right|$ of each finger as well as resultant force $\left|\sum_{i} \boldsymbol{f}_{z_{i}}\right|$ are measured for controlling finger positions. $|\boldsymbol{W}|$ is updated in every stable grasping state.

\section{Strategy for Manipulating Deformable Heavy Object}

When the weight of grasped deformable object increases (for instance, by pouring water into a grasped container), the robotic gripper should either apply larger force to prevent the object from sliding, which may result in crushing the object; or just release the object immediately. However, humans can still hold the deformable heavy objects (even with dynamic centers of mass) by rotating them and changing their orientations/positions. Although the desired orientation of the grasped object is changed, the object may be safely grasped without increasing the applied force. Considering an object of weight $|\boldsymbol{W}|$ is grasped stably, and all tangential forces on the contact surface $\left(\boldsymbol{f}_{T_{i}}\right)$ are directed toward $-Z_{W C F}$, i.e. $Z_{W C F}$ parallels $Z_{S C F}$, and then the object is rotated by an angle $\theta(\theta \in[0, \pi / 2])$. If the grasp is stable, exerted force should satisfy:

$$
\begin{gathered}
\sum_{i} \boldsymbol{f}_{N_{i}}+\sum_{i} \boldsymbol{f}_{T_{i}}+\boldsymbol{W}=\mathbf{0} \\
\sum_{i}\left|\boldsymbol{f}_{T_{i}}\right| \leq \sum_{i} \mu_{i}\left|\boldsymbol{f}_{N_{i}}\right| .
\end{gathered}
$$

As the target object is grasped stably, the resultant force $\left|\sum_{i} \boldsymbol{f}_{T_{i}}\right|$ (i.e. the friction) is able to balance the weight component $|\boldsymbol{W}| \cos \theta$ which is tangential to the contact surface; however, if the resultant tangential force is not able to compensate this weight component (for each finger, the maximum $\left|\boldsymbol{f}_{T_{i}}\right|$ available is provided by $\mu_{i}\left|\boldsymbol{f}_{N_{i}}\right|$, Eq. 2), slip occurs. Therefore, slip can be stopped either by increasing $\left|\sum_{i} \boldsymbol{f}_{N_{i}}\right|$, i.e. enhancing the upper bound of tangential force, or by reducing $|\boldsymbol{W}| \cos \theta$, which equals increasing $\theta$ in $[0, \pi / 2]$. The former approach has been applied for slip prevention in previous research [17]. However, since normal forces should be constrained to prevent large deformation (section III-A), we suggest exploiting the latter strategy, i.e. reducing weight component $|\boldsymbol{W}| \cos \theta$ by rotating, especially for the manipulation of deformable objects. This approach can be applied to control the gripper by following the procedure in Algorithm 2 after the initialization and slip detection procedures in Algorithm 1.

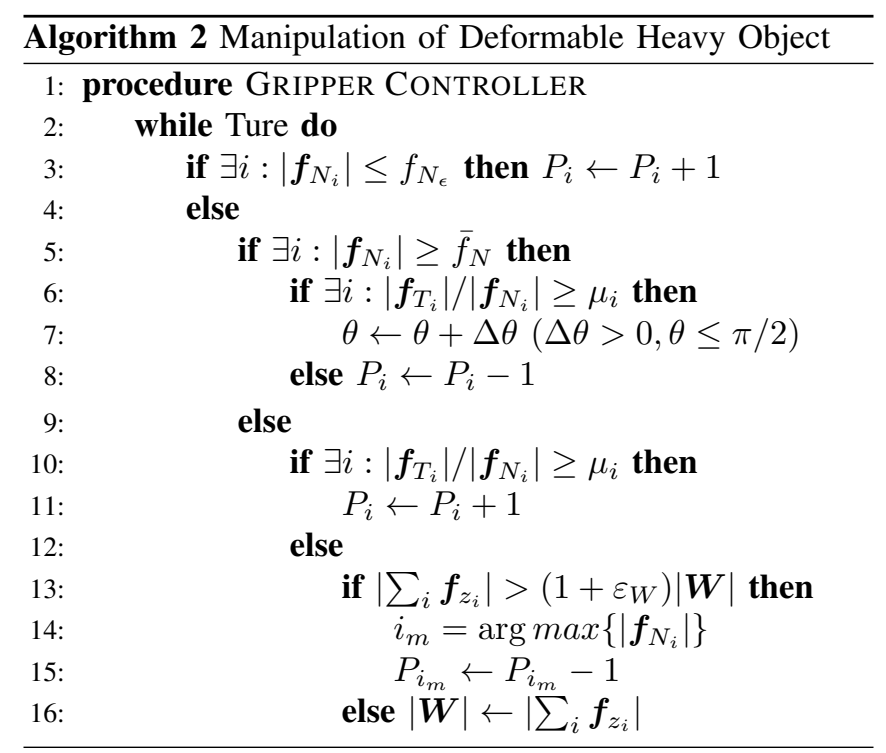

\section{EXPERIMENTS}

The robotic system performed four groups of experiments. In the first three groups of experiments, the task was to grasp and then lift the deformable objects by applying three different grasping strategies, i.e. gripper's built-in adaptive grasping mechanism, manipulation with minimal grasping force, and our proposed framework. Furthermore, to evaluate and compare the performances of all grasping strategies, the 
weight of the grasped object was increased by either pouring rice or water inside, and then the object was manipulated and rotated by $\pm 2 \pi / 3$. The last group of experiments was carried out to evaluate the performance of the proposed strategy for manipulating deformable heavy objects. In this work, the UR10 robotic arm, the Robotiq gripper, and the OptoForce sensors were controlled in the framework of ROS (Robot Operating System). Tactile signals were sampled at a frequency of $333 \mathrm{~Hz}$ and then processed by a $15 \mathrm{~Hz}$ low-pass filter, while the gripper was controlled at $50 \mathrm{~Hz}$.

\section{A. Gripper's Built-in Adaptive Grasping Mechanism}

In this scenario, the task of the gripper was to grasp the experimental objects using the built-in adaptive grasping mechanism without tactile feedback. When the gripper grasps an object, each finger stops moving as soon as the exerted force exceeds $15 \mathrm{~N}$, which is the minimum force can be detected by the gripper. Exploiting the built-in adaptive grasping approach, the gripper managed to grasp all the experimental objects without any slip; however, the objects were gravely deformed due to the large grasping force (see Fig. 2).

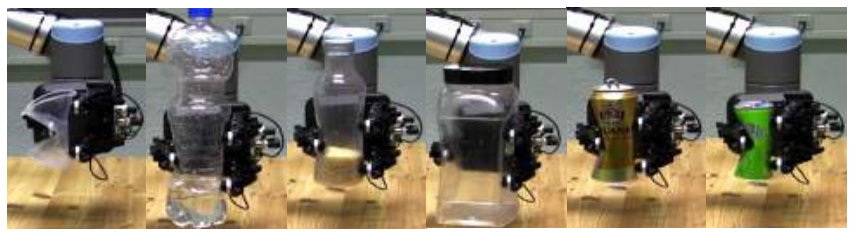

Fig. 2. Grasping deformable objects using gripper's built-in adaptive grasping mechanism.

\section{B. Minimum Force Grasping}

In this experiment, the minimum grasping force strategy was used (see Algorithm 1). The gripper first kept closing its fingers till the target object was grasped by a small contact force $f_{N_{\epsilon}}$, with $0.2 \mathrm{~N} \leq f_{N_{\epsilon}} \leq 0.5 \mathrm{~N}$, according to target object's stiffness. As soon as the first slip was stopped, the minimum grasping force for each finger was determined (Algorithm 1-18, where $\delta=5 \%$ and $\Delta t=300 \mathrm{~ms}$ ). After the target object was grasped (see Fig. 3), we either increased the weight of grasped object manually, or rotated it using robotic system by $\pm 2 \pi / 3$. Experimental results in Fig. 3 show that the robotic system using minimum grasping force strategy is able to grasp the experimental objects with different stiffnesses and textures without large deformation. Nevertheless, objects slid out of the gripper. During manipulation, slips were caused by either weight increment or changes of the centers of mass, which the gripper could neither detect nor prevent. Therefore, the minimum grasping force can prevent the deformation of grasped objects to a great extent; however, it is not capable of preventing grasped objects from slipping in dynamic manipulation tasks.

\section{Evaluation of the Proposed Grasping Strategy}

The task was to manipulate the experimental objects safely as described in the previous part. However, different from

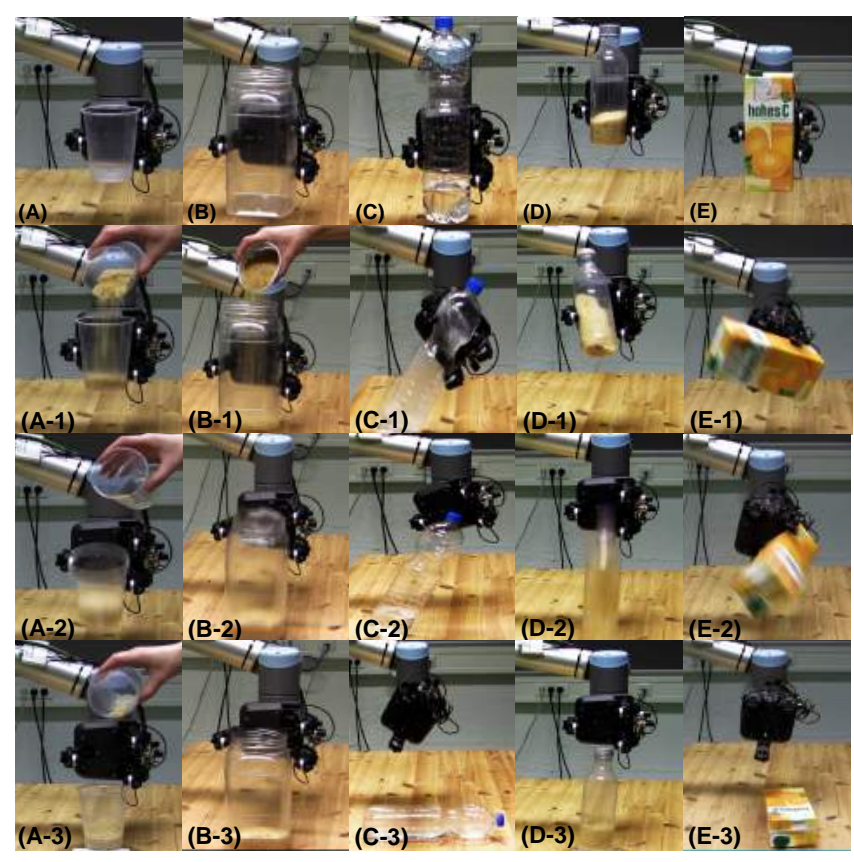

Fig. 3. Manipulation of deformable objects with different characteristics, such as stiffness, surface texture, and center of mass, using minimum grasping force.

section IV-B, the robotic system was controlled by our proposed slip correction and grip force regulation framework (Algorithm 1) in this scenario. The upper bound of normal force was set to $2 \mathrm{~N} \leq f_{N} \leq 5 \mathrm{~N}$ in order to reduce local deformation, according to each target object's stiffness. In order to tune the relative positions of the three fingers, the margin of resultant force was set in $Z_{W C F}$ direction w.r.t. the estimated weight to $\varepsilon_{W}=40 \%$, according to the research result that the human central nervous system constantly adjusts the grip force with a margin of $10 \%-40 \%$ [18]. In this experiment, the gripper closed its fingers for one position count once slip was detected, while the weight of the grasped object kept increasing. Hence, the target object was grasped stably without any slip during the entire process (see Fig. 4A). In case of manipulation/rotation, the grasped object with dynamic center of mass ( $\mathrm{CoM})$ was manipulated stably by the robotic system, and the deformation of the object's surface was minimized as much as possible (see Fig. 4). Normal and tangential force signals recorded in one of the dynamic manipulation experiments are plotted in Fig. 5. In this scenario, a deformable bottle filled with $300 \mathrm{~mL}$ water was rotated (see Fig. 4-B). After initialization procedure (see Fig. 5-A), the robotic system grasped the bottle and lifted it up, till the first slip happened and was stopped (see Fig. 5B). Then the robotic system started rotating the half-bottle of water by $\pm 2 \pi / 3$ for two times (see Fig. 5-C and 5-D). In the meantime, the gripper was controlled to correct slips and to regulate grasping force, in order to prevent the grasped bottle from sliding (especially as the abrupt changes of CoM happened) and large deformation. Compared to the minimum grasping force based manipulation, gripper reacted promptly either to correct slip or to regulate large normal force. 


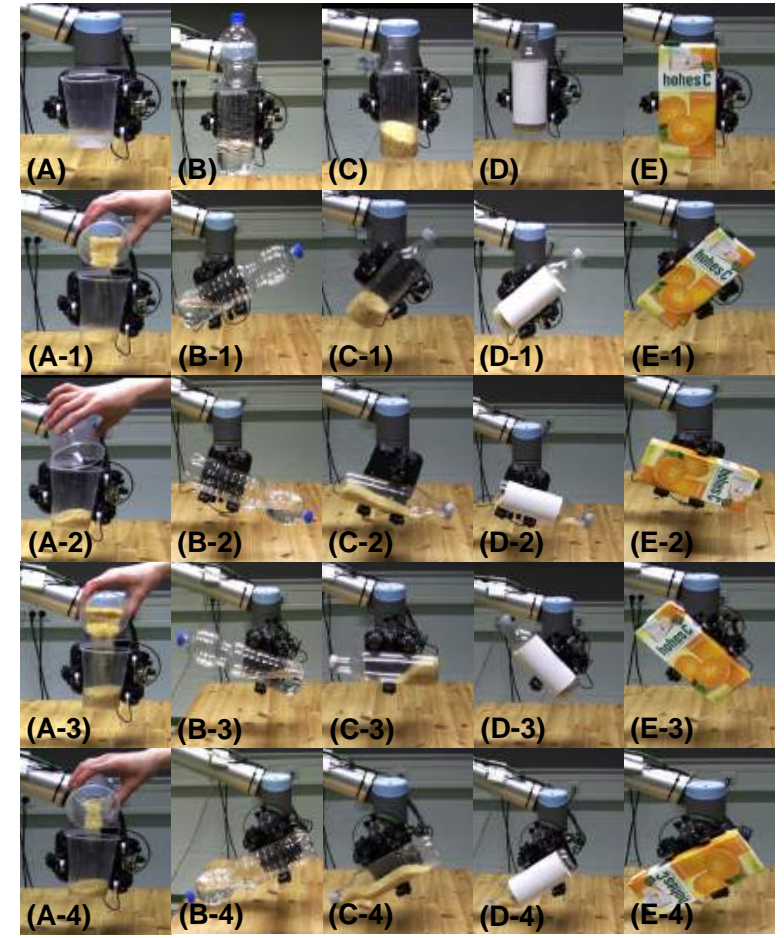

Fig. 4. Manipulation of deformable objects with different characteristics, such as stiffness, surface texture, and center of mass, using Algorithm 1.
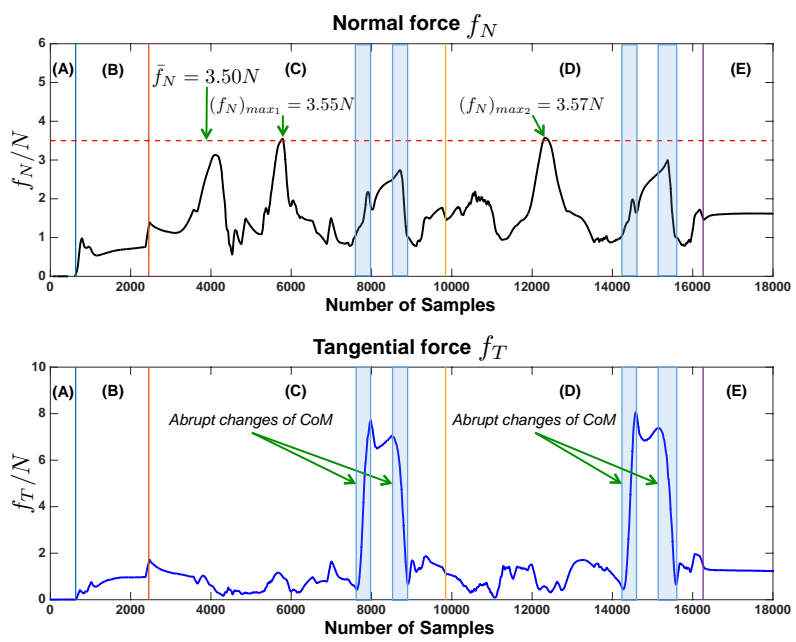

Fig. 5. Normal force and tangential force exerted by one of the fingers (here finger A) recorded while manipulating a deformable bottle of $300 \mathrm{~mL}$ water.

\section{Comparison of Three Different Grasping Approaches}

In order to measure the deformation extent of grasped objects, the difference between the average positions of fingers from the opposite sides $\left(P_{1}\right.$ and $\left.\left(P_{2}+P_{3}\right) / 2\right)$ was calculated as an indication. Hence, a large finger position difference indicates a small deformation extent and vice versa. Maximum deformation values were recorded for experimental objects during manipulations by using both the adaptive grasping mechanism and the proposed gripper controller, respectively (see Fig. 7-A); while Fig. 7-B shows the deformation extent compared to the original size in percentage. Although the grasped objects were severely deformed in experiments using

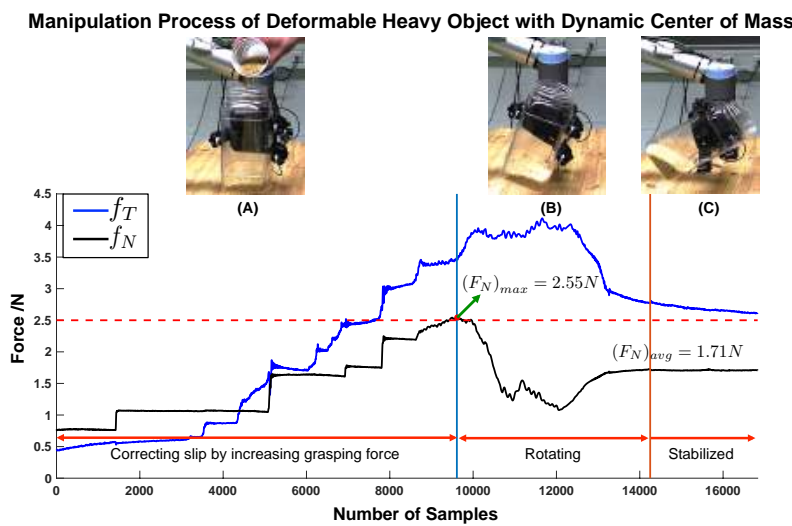

Fig. 6. Manipulating a deformable heavy jar by applying Algorithm 2. Plots show the recorded normal and tangential forces exerted by (here finger B ) during the manipulation process.

the adaptive grasping mechanism, deformation was largely reduced by taking advantage of the proposed gripper controller.

\section{E. Evaluation of the Proposed Manipulation Strategy for Deformable Heavy Objects}

The objective of this experiment was to evaluate the performance of the proposed strategy for manipulating deformable heavy objects. The positive rotation direction $(\theta>$ 0 ) of the gripper was specified as from finger A to finger B, through finger $\mathrm{C}$. As $\theta$ increases from 0 to $\pi / 2$, the resultant force $\left|\sum_{i} \boldsymbol{f}_{T_{i}}\right|$ required to balance the weight component $|\boldsymbol{W}| \cos \theta$ reduces. The difference between normal forces $\left(f_{N_{i}}\right)$ exerted by fingers from opposite sides should compensate $|\boldsymbol{W}| \sin \theta$, which increases during rotation. For the three-finger gripper, the condition for normal forces in stable state can be described as:

$$
|| \boldsymbol{f}_{N_{2}}|+| \boldsymbol{f}_{N_{3}}|-| \boldsymbol{f}_{N_{1}}||=|\boldsymbol{W}| \sin \theta
$$

As $\theta$ gets close to $\pi / 2$, both $\boldsymbol{f}_{N_{2}}$ and $\boldsymbol{f}_{N_{3}}$ approach $(|\boldsymbol{W}|-$ $\left.\left|\boldsymbol{f}_{N_{1}}\right|\right) / 2$. In this experiment, the task of the robotic system was to grasp the deformable plastic jar safely, while exploiting the proposed strategy in Algorithm 2. After initialization and slip detection procedures, the empty jar was grasped with the determined minimum force. Then the weight of the grasped object was continuously increased by pouring rice into it. During this process, the gripper closed its fingers as soon as slip was detected (see Fig. 6-A). As the jar became heavier, the exerted normal force exceeded its upper bound (see Fig. 6, around 9500th sample of sensor signal), which means slip could not be stopped by increasing normal force any more. Thus, the gripper started rotating target object while tuning the relative positions of each finger simultaneously to find an equilibrium pose (see Fig. 6-B). Afterwards, tangential force reduced, indicating the stop of slip; and normal force converged, showing a stable grasping state (see Fig. 6-C). Fig. 6 shows that the robotic system managed to grasp and manipulate the deformable heavy jar successfully. It is worthwhile to mention that the position 


\section{Comparison of Grasping Deformation}
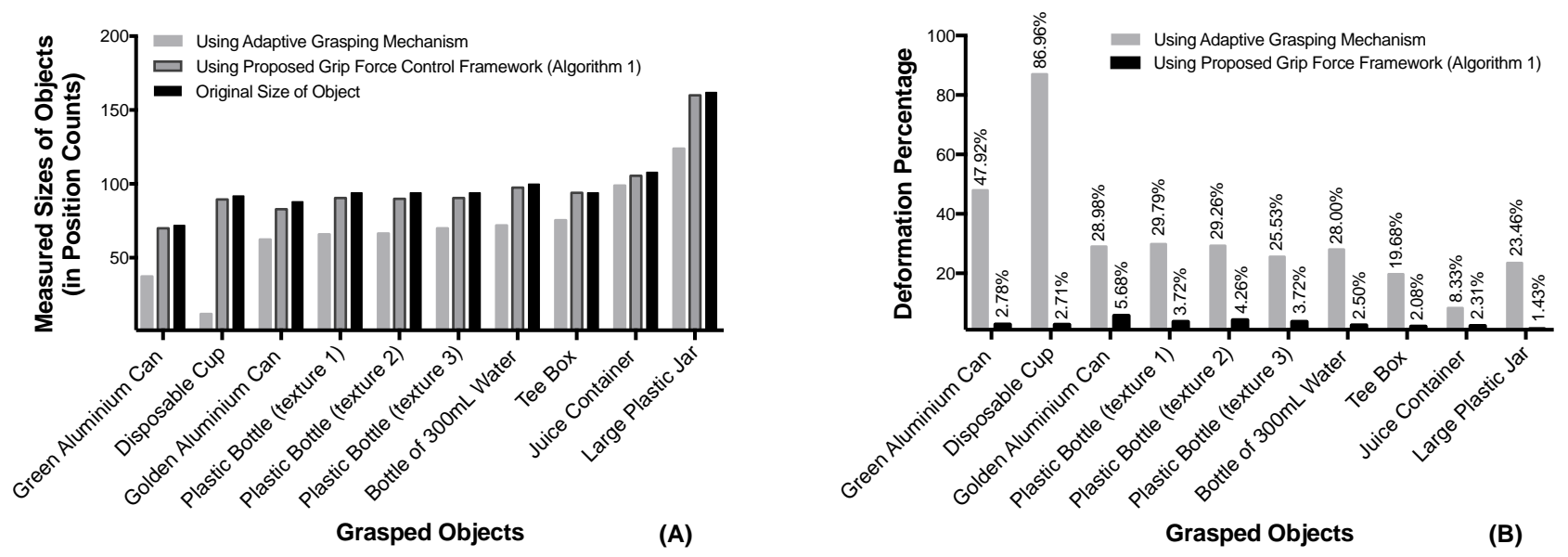

Fig. 7. Comparison of deformation extents of grasped objects, using gripper's built-in adaptive grasping mechanism and the proposed slip correction and force regulation framework, respectively.

of each finger is kept being adjusted during the rotation process, in order to avoid the occurrence of re-orientation phenomenon, which means the grasped object has reached a new position in hand because of slippage.

\section{Conclusions And Future Work}

Approaches proposed in this work have several advantages over prior work. The approach used in this study can be executed online, and does not require any prior knowledge of the contact surface (e.g. friction coefficient). Since our proposed approach does not rely on frequency analysis, it is insusceptible to the vibration signals generated by the robotic system during manipulation, and also robust to external disturbances. In addition, our proposed framework is able to control multiple-fingers of the gripper individually in real time, and is independent of the properties of the grasped object, such as stiffness, surface texture, and center of mass. In the future, a low-level control (e.g. force control or torque control) of the gripper is expected for a more compliant behavior, which is not available currently for a finger position controller. Moreover, the performance could be improved by embedding the computation of break-away ratio into our framework.

\section{ACKNOWLEDGMENT}

This work is supported by the European Commission under grant agreements PITN-GA-2012-317488-CONTEST. Many thanks to OptoForce Ltd. for providing tactile sensors for this study.

\section{REFERENCES}

[1] D. A. Bennett, S. A. Dalley, and et al., "A multigrasp hand prosthesis for providing precision and conformal grasps," IEEE ASME Transactions on Mechatronics, vol. 20, no. 4, pp. 1697-1704, 2015.

[2] M. Kaboli, P. Mittendorfer, V. Hugel, and G. Cheng, "Humanoids learn object properties from robust tactile feature descriptors via multi-modal artificial skin," in IEEE-RAS International Conference on Humanoid Robots, pp. 187-192, 2014.

[3] M. Kaboli, T. D. L. Rosa, R. Walker, and G. Cheng, "In-hand object recognition via texture properties with robotic hands, artificial skin, and novel tactile descriptors," in IEEE-RAS International Conference on Humanoid Robots, pp. 1155-1160, 2015.
[4] M. Kaboli, R. Walker, and G. Cheng, "Re-using prior tactile experience by robotic hands to discriminate in-hand objects via texture properties," in IEEE International Conference on Robotics and Automation, pp. 2242-2247, 2016.

[5] M. Kaboli and G. Cheng, "Dexterous hands learn to re-use the past experience to discriminate in-hand objects from the surface texture," in 33rd Annual Conference of the Robotics Society of Japan, 2015.

[6] N. Yogeswaran and et al., "New materials and advances in making electronic skin for interactive robots," Advanced Robotics, vol. 29, no. 21, pp. 1359-1373, 2015.

[7] M. Kaboli, A. Long, and G. Cheng, "Humanoids learn touch modalities identification via multi-modal robotic skin and robust tactile descriptors," Advanced Robotics, vol. 29, no. 21, pp. 1411-1425, 2015.

[8] E. D. Engeberg and S. G. Meek, "Adaptive sliding mode control for prosthetic hands to simultaneously prevent slip and minimize deformation of grasped objects," IEEE ASME Transactions on Mechatronics, vol. 18, no. 1, pp. 376-385, 2013.

[9] S. Teshigawara, K. Tadakuma, A. Ming, M. Ishikawa, and M. Shimojo, "High sensitivity initial slip sensor for dexterous grasp," in IEEE International Conference on Advanced Robotics, 2010.

[10] M. Schöpfer, C. Schürmann, M. Pardowitz, and H. Ritter, "Using a piezo-resistive tactile sensor for detection of incipient slippage," in International Symposium onRobotics and 6th German Conference on Robotics (ROBOTIK), pp. 1-7, VDE, 2010.

[11] D. P. Cotton, P. Chappell, A. Cranny, et al., "A novel thick-film piezoelectric slip sensor for a prosthetic hand," IEE Sensors Journal, vol. 7, no. 5, pp. 752-761, 2007.

[12] F. Kobayashi, H. Kanno, H. Nakamoto, and F. Kojima, "Slip based pick-and-place by universal robot hand with force/torque sensors," in IEEE Symposium on Robotic Intelligence In Informationally Structured Space, pp. 1-4, 2014.

[13] C. Melchiorri, "Slip detection and control using tactile and force sensors," IEEE ASME transactions on mechatronics, vol. 5, no. 3, pp. 235-243, 2000.

[14] B. Heyneman and M. R. Cutkosky, "Slip interface classification through tactile signal coherence," in IEEE International Conference on Intelligent Robots and Systems, pp. 801-808, 2013.

[15] J. Reinecke, A. Dietrich, F. Schmidt, and M. Chalon, "Experimental comparison of slip detection strategies by tactile sensing with the biotac $($ $)$ on the dlr hand arm system," in IEEE International Conference on Robotics and Automation, pp. 2742-2748, 2014.

[16] Z. Su and et al., "Force estimation and slip detection/classification for grip control using a biomimetic tactile sensor," in IEEE International Conference on Humanoid Robots, pp. 297-303, 2015.

[17] N. Wettels and et al., "Grip control using biomimetic tactile sensing systems," IEEE/ASME Transactions On Mechatronics, vol. 14, no. 6, pp. 718-723, 2009.

[18] L. Roberts, G. Singhal, and R. Kaliki, "Slip detection and grip adjustment using optical tracking in prosthetic hands," in IEEE Annual International Conference of Engineering in Medicine and Biology Society, pp. 2929-2932, 2011. 